\title{
Reações adversas aos antirretrovirais apresentadas pelos portadores de HIV
}

\section{Adverse reactions to antiretrovirals presented by HIV patients: a scientific}

Reacciones adversas a antirretrovirales presentadas por pacientes con VIH: una revisión científica

Higo José Neri da Silva

ORCID: https://orcid.org/0000-0003-2089-4147

Faculdade de Tecnologia de Teresina, Brasil

E-mail: higoneri@gmail.com

Pauliana Texeira da Silva

ORCID: https://orcid.org/0000-0003-0358-8025 Faculdade SEVEN, Brasil

paulianateixeiras2513@gmail.com

Ana Maria Castro Ferreira

ORCID: https://orcid.org/0000-0003-4378-2893

Universidade de Campinas, Brasil

E-mail: castroferreiraana@gmail.com

Wallyson André dos Santos Bezerra

ORCID: https://orcid.org/0000-0002-9151-9654

Universidade Federal do Maranhão, Brasil

E-mail: w_andre168@hotmail.com

Denilson de Araújo e Silva

ORCID: https://orcid.org/0000-0001-5401-3462

Centro Universitário Uninovafapi, Brasil

E-mail: bmdenilsonaraujo@outlook.com

Mariel Rodrigues de Campos

ORCID: https://orcid.org/0000-0002-7253-2212

Centro Universitário Maurício de Nassau, Brasil

E-mail: maryel_r@hotmail.com

Antonia Luzia Lima do Nascimento

ORCID: https://orcid.org/0000-0001-9740-5669

Universidade Federal do Rio Grande do Sul, Brasil

E-mail: antonialuzialimaa@gmail.com

Bruna Layra Silva

ORCID: https://orcid.org/0000-0002-7385-0095 Universidade Estadual do Piauí, Brasil

E-mail: biobiomed.brunalayra@gmail.com

Deylane Menezes Teles e Oliveira

ORCID: https://orcid.org/0000-0002-5516-9167

Universidade Federal do Piauí, Brasil E-mail: deylane.teles@gmail.com

Alice Maria de Castro Ribeiro

ORCID: https://orcid.org/0000-0002-3506-8772

Instituto Federal do Piauí, Brasil

E-mail: alyce-mcr@hotmail.com

Amanda Miranda da Silva

ORCID: https://orcid.org/0000-0001-6476-4809

Universidade Federal do Piauí, Brasil E-mail:miraanda.sa@gmail.com 


\title{
Resumo
}

Os portadores de HIV iniciam seus tratamentos no do Sistema Único de Saúde (SUS) através da Assistência da Estratégia da Saúde da Família (ESF) e esta, por sua vez, coordena todos os cuidados prestados e o encaminhamento do paciente a uma unidade de referência para aconselhamento, acompanhamento e controle da infecção de forma integral e resolutiva. Contudo esperamos que este trabalho contribua para compreendermos os tipos de reações adversas ocorridas pelos usuários dos medicamentos para tratamento do HIV. Foram utilizadas todas as publicações que possuírem dados de tratamentos farmacológicos para HIV, dentre a determinada data (2019-2020). Os dados foram coletados utilizando as bases cientificas: Literatura Latino-Americana e do Caribe em Ciências da Saúde (LILACS), National Library of medicine (PUBMED), PERIODICOS, SCIENCE DIRECT, Biblioteca Virtual em saúde - (BVS), Scientific Eletronic Library Online (SCIELO) e Cochrane Library; e as bases tecnológicas: World Intellectual Property Organization (WIPO) e Instituto Nacional de Propriedade Industrial (INPI). Utilizamos como descritores: REAÇÕES ADVERSAS AOS ANTIRRETROVIRAIS, REAÇÕES ADVERSAS AOS ANTIRRETROVIRAIS EM GESTANTES e ANTIRRETROVIRAIS. O idioma de publicação estava em português, inglês e espanhol, atendendo a uma série histórica de pelo menos cinco anos. Foi observado 208.425 artigos nas bases cientificas. Esse tratamento do vírus não cura por conta do acumulo persistente de células infectadas latentemente. Mesmo com sintomas leves, podem levar a uma diminuição da qualidade de vida do portador, assim necessário mais pesquisas sobre o tema. Devemos lembrar que a qualidade de vida deles, impacta diretamente na nossa sociedade e, portanto, importa a todos.

Palavras-chave: Antirretrovirais; Efeito colateral; HIV; Doença negligenciada; Saúde pública.

\begin{abstract}
HIV patients start their treatments in the Unified Health System (SUS) through the Family Health Strategy Assistance (ESF) and this, in turn, coordinates all care provided and the referral of the patient to a reference unit for counseling, monitoring and control of infection in an integral and resolutive way. However, we hope that this work will contribute to understanding the types of adverse reactions that have occurred by users of HIV medications. All publications that have data on pharmacological treatments for HIV were used, from a certain date (2019-2020). Data were collected using the scientific databases: Latin American and Caribbean Literature in Health Sciences (LILACS), National Library of medicine (PUBMED), PERIODICOS, SCIENCE DIRECT, Virtual Health Library ( VHL), Scientific Electronic Library Online (SCIELO) and Cochrane Library; and the technological bases: World Intellectual Property Organization (WIPO) and National Institute of Industrial Property (INPI). We use as descriptors: ADVERSE REACTIONS TO ANTIRETROVIRAL DRUGS, ADVERSE REACTIONS TO ANTIRETROVIRAL DRUGS IN PREGNANT WOMEN and ANTIRETRORETROVIRAL. The publishing language was in Portuguese, English and Spanish, attending a historical series of at least five years. A number of articles were observed on the scientific database. This treatment of the virus does not cure because of the persistent accumulation of latentemente infected cells. Even with mild symptoms, they can lead to a decrease in the quality of life of the carrier, thus necessary more research on the subject. We must remember that their quality of life directly impacts our society and therefore matters to everyone.
\end{abstract}

Keywords: Antiretrovirals; Side effect; HIV; Neglected disease; Public health.

\section{Resumen}

Los pacientes con VIH inician sus tratamientos en el Sistema Unificado de Salud (SUS) a través de la Ayuda a la Estrategia de Salud Familiar (FSE) y esto, a su vez, coordina toda la atención prestada y la derivación del paciente a una unidad de referencia para el asesoramiento, seguimiento y control de la infección de forma integral y resolutiva. Sin embargo, esperamos que este trabajo contribuya a comprender los tipos de reacciones adversas que han producido 
los usuarios de medicamentos contra el VIH. Todas las publicaciones que tienen datos sobre tratamientos farmacológicos para el VIH se utilizaron, a partir de una fecha determinada (2019-2020). Los datos fueron recopilados utilizando las bases de datos científicas: Literatura Latinoamericana y del Caribe en Ciencias de la Salud (LILACS), Biblioteca Nacional de Medicina (PUBMED), PERIODICOS, SCIENCE DIRECT, Biblioteca Virtual de Salud (VHL), Biblioteca Electrónica Científica en Línea (SCIELO) y Biblioteca Cochrane; y las bases tecnológicas: Organización Mundial de la Propiedad Intelectual (OMPI) y Instituto Nacional de la Propiedad Industrial (INPI). Utilizamos como descriptores: REACCIONES ADVERSAS A MEDICAMENTOS ANTIRRETROVIRALES, REACCIONES ADVERSAS A MEDICAMENTOS ANTIRRETROVIRALES EN MUJERES EMBARAZADAS Y ANTIRRETRORETROVIRALES. El idioma editorial estaba en portugués, inglés y español, asistiendo a una serie histórica de al menos cinco años. Se observaron varios artículos en la base de datos científica. Este tratamiento del virus no se cura debido a la acumulación persistente de células infectadas por latentemente. Incluso con síntomas leves, pueden conducir a una disminución en la calidad de vida del portador, por lo tanto, es necesario más investigación sobre el tema. Debemos recordar que su calidad de vida afecta directamente a nuestra sociedad y, por lo tanto, importa a todos. Palabras clave: Antirretrovirales; Efecto colateral; HIV; Enfermedad desatendida; Salud pública.

\section{Introdução}

A Síndrome da Imunodeficiência Adquirida (AIDS) tem sido intensa e amplamente estudada, desde os primeiros casos diagnosticados, na década de 70. No entanto, mesmo com o avanço da ciência e da tecnologia, especialistas alertam que a doença ainda é incurável e que a cada dia mais pessoas se contaminam no Brasil e no mundo (Brasil, 2018).

Há uma estimativa mundial que mais de 35 milhões de pessoas vivam com o Vírus da Imunodeficiência Humana (HIV), um grave problema de saúde pública, que resultou em mais de 1,5 milhão de óbitos entre 1980 e 2013, despertando a atenção das autoridades e profissionais de saúde para promoção e combate dessa epidemia, no tocante às medidas de prevenção, diagnóstico, tratamento e reabilitação dos indivíduos (Silva et al, 2016; Suto et al, 2017).

No Brasil, a lei federal no 913 de 13 de novembro de 1996, garante o acesso universal e gratuito ao tratamento antirretroviral e, ao longo do tempo, também foi disponibilizado à população os exames de monitoramento laboratorial, hoje facilitado pelo uso de testes rápidos, que detectam a presença de anticorpos contra o HIV em menos de 30 minutos, têm custo baixo e são de simples aplicação e interpretação (Brasil, 1996; Alochio et al, 2016; Oliveira et al, 2018).

Os portadores de HIV iniciam seus tratamentos no do Sistema Único de Saúde (SUS) através da Assistência da Estratégia da Saúde da Família (ESF) e esta, por sua vez, coordena todos os cuidados prestados e o encaminhamento do paciente a uma unidade de referência para aconselhamento, acompanhamento e controle da infecção de forma integral e resolutiva (Silva et al, 2017).

Com a aplicação da zidovudina (AZT) vem se reduzindo os riscos de infecção pelo HIV após exposição ocupacional percutânea em uma faixa de $81 \%$ dos casos. A forma correta de se utilizar a medicação favorece o restabelecimento do paciente de forma segura e eficaz ao resto da sua vida. No entanto, um número importante de portadores apresenta algumas reações adversas, fazendo com que os soropositivos apresentem mal-estar. Mas nem todos soropositivos apresentam esses efeitos colaterais, alguns sentem esses efeitos somente no início da medicação com o decorrer do tempo o organismo tem uma boa aceitabilidade (Brasil, 2013; Alverca et al, 2017).

Esses remédios impedem o vírus de se reproduzir nas células CD4 e, como consequência, a quantidade de vírus no sangue cai para um nível indetectável. Com relação aos coquetéis anti-HIV podem causar danos aos rins, fígado, ossos, estômago e intestino e distúrbios neuropsiquiátricos. Podem também modificar o metabolismo, provocando lipodistrofia que é definida por uma mudança na distribuição de gordura pelo corpo, havendo o aparecimento da diabetes (Huffpost, 2017).

É inquestionável a suma importância da implementação de políticas públicas voltadas para o tratamento de pessoas convivendo com HIV\AIDS, devendo-se abordar o conjunto de aspectos compreendidos na caracterização assistencial farmacêutica. É neste momento que o acompanhamento do farmacêutico nas consultas periódicas aprazadas, poderá esclarecer dúvidas a respeito de seus medicamentos com o objetivo de promover o uso seguro das medicações, a partir da avaliação 
farmacoterápica. Visto que, a maioria dos pacientes não compreende que as interações medicamentosas podem ocasionar riscos no decorrer de seu tratamento. Desta forma, a informação clara e objetiva se torna presente, quando o paciente começa a entender e respeitar os horários e vias de administração, bem como as doses prescritas pelo médico responsável, assim como o malefício que pode ocasionar à sua saúde caso haja a interrupção, troca do hábito continua ou esporádico da automedicação (Fonseca et al, 2018).

A evolução nas pesquisas e estudos dos medicamentos Anti-HIV trazem melhorias aos pacientes desde níveis tóxicos, potência terapêutica, tolerância por parte do paciente até a quantidade de comprimidos para permitir a supressão viral durável para a maioria dos infectados.

O polimorfismo genético também tem sido um problema para a farmacogenética, pois a existência de diferentes tipos de alelos de um mesmo gene implica mudanças nas sequências genéticas entre seres o que pode levar a reações adversas aos medicamentos anti-HIV. A resposta ao tratamento da infecção pelo HIV é um fenômeno complicado podendo ocasionar deficiências nas terapias e, muitas vezes, limitado pela ocorrência de níveis tóxicos agudos ou crônicos ou de resistência viral e interações medicamentosas. As intervenções precursoras contra esta infecção foram definidas pela intransigência por parte dos indivíduos às altas doses do único fármaco disponível na época, a Zidovudina - AZT (Silva et al, 2013).

$\mathrm{O}$ ano de 2020 tem sido marcado por outra preocupação mundial, devido à disseminação de uma doença causada pelo Coronavírus SARS-CoV-2, que, se acordo com Organização Mundial da Saúde (World Health Organization - WHO), pode causar síndrome respiratória aguda grave, podendo causar lesões nos sistemas respiratório, hepático, gastrointestinal e neurológico. O que pode ser ainda mais grave em portadores de HIV/AIDS por sua condição de imunodeficiência, ocasionando atenção redobrada pelos profissionais de saúde (Brasil, 2020; Unaids, 2020).

Esta pesquisa é de suma importância para que todos os usuários que necessitem de apoio e informações do farmacêutico em ambiente hospitalar ou áreas afins compreendam que o acompanhamento deste profissional aos portadores de HIV tem efeito considerável e significativo para possibilitar, facilitar e favorecer o auxílio de resolutividade, sanar dúvidas que possam favorecer a diminuição das reações adversas nas terapias medicamentosas. Diante do exposto esperamos que este projeto de pesquisa colabore para a compreensão das medidas que envolvam a prevenção e complicações das reações adversas geradas pelo o uso das medicações antirretrovirais, em especial a AZT, além de apontar lacunas do conhecimento que precisam ser preenchidas com a realização de novos estudos, permitindo assim, a disseminação de conhecimento para acadêmicos da área, assim como, a população de um modo geral.

Contudo esperamos que este trabalho contribua para compreendermos os tipos de reações adversas ocorridas pelos usuários dos medicamentos para tratamento do HIV.

\section{Metodologia}

Trata-se de uma pesquisa cientifica de objetivo descritivo e explicativo com abordagem qualitativa. Essa pesquisa busca através de publicações cientificas descrever seus resultados, explicando suas causas e efeitos. Sua abordagem implica que tudo o que for realizado será qualificado para melhor demonstração dos resultados obtidos pela pesquisa. Onde foi utilizado programa Prisma para melhor distribuição e interpretação dos dados estatísticos (Kauark, 2010; Pereira et al, 2018).

Foram utilizadas todas as publicações que possuíram dados de tratamentos farmacológicos para HIV, publicações cientificas oficiais da Organização Mundial da Saúde (World Health Organization - WHO) e governamentais, dentre a determinada data (2019-2020).

Os dados foram coletados utilizando as bases cientificas: Literatura Latino-Americana e do Caribe em Ciências da Saúde (LILACS), National Library of medicine (PUBMED), Periodicos, Science Direct, Biblioteca Virtual em saúde - (BVS), Scientific Eletronic Library Online (SCIELO) e Cochrane Library. 
Os artigos foram selecionados por meio de critérios dialogados entre os pesquisadores que seja de total relevância para o estudo a ser pesquisado. Os artigos atenderam ao tema proposto que ofereceram informações sobre as reações adversas apresentadas pelos portadores com HIV. Utilizamos como descritores as nomenclaturas: reações adversas aos antirretrovirais, reações adversas aos antirretrovirais em gestantes e antirretrovirais. As publicações estavam nos idiomas: português, inglês e espanhol, àqueles que atenderam a uma série histórica de pelo menos cinco anos, ou seja, todos os artigos respeitaram a sua temporalidade de dados.

Foram excluídos todos os artigos em outros idiomas não aceitos pelos pesquisadores, além de livros, capítulos de livros, manuais, resumos de anais, revisões de literatura, dissertações, teses, monografias e estudos duplicados.

A pesquisa não possui muitos riscos, pois todos os dados serão ofertados pelas bases cientificas, onde não houve contato nenhum com os indivíduos infectados e não foram divulgados dados pessoais dos mesmos. A pesquisa foi apenas análise de dados para demonstração do assunto tratado.

\section{Resultados e Discussão}

Na Tabela a seguir nós observamos a quantidade de artigos encontrados nas bases cientificas Cochrane Library, SCIELO, LILACS, PUBMED, PERIODICOS, SCIENCE DIRECT, Biblioteca Virtual em saúde onde em geral foram obtidos 208.425 artigos científicos com as palavras chaves Reações Adversas Aos Antirretrovirais, Reações Adversas Aos Antirretrovirais Em Gestantes E Antirretrovirais, nos idiomas inglês, português e espanhol. Sendo que apenas 947 eram em português, de início já mostrando a importância em mais pesquisas no nosso idioma.

Tabela 1. Quantidade de artigos encontrados de acordo com cada palavra chave.

\begin{tabular}{|c|c|c|c|}
\hline Bases cientificas & Descritor 1 & Descritor 2 & Descritor 3 \\
\hline & $\begin{array}{c}\text { Reações adversas aos } \\
\text { antirretrovirais }\end{array}$ & $\begin{array}{l}\text { Reações adversas aos } \\
\text { antirretrovirais em gestantes }\end{array}$ & Antirretrovirais \\
\hline LILACS & $\begin{array}{l}2 \text { Inglês } \\
1 \text { Português } \\
4 \text { Espanhol }\end{array}$ & $\begin{array}{l}0 \text { Inglês } \\
0 \text { Português } \\
0 \text { Espanhol }\end{array}$ & $\begin{array}{l}67 \text { Inglês } \\
66 \text { Português } \\
57 \text { Espanhol }\end{array}$ \\
\hline PUBMED & $\begin{array}{l}2317 \text { Inglês } \\
0 \text { Português } \\
5 \text { Espanhol }\end{array}$ & $\begin{array}{l}55 \text { Inglês } \\
0 \text { Português } \\
0 \text { Espanhol }\end{array}$ & $\begin{array}{l}125427 \text { Inglês } \\
5 \text { Português } \\
9 \text { Espanhol }\end{array}$ \\
\hline PERIODICOS & $\begin{array}{l}13 \text { Inglês } \\
11 \text { Português } \\
9 \text { Espanhol }\end{array}$ & $\begin{array}{l}2 \text { Inglês } \\
0 \text { Português } \\
1 \text { Espanhol }\end{array}$ & $\begin{array}{l}223 \text { Inglês } \\
198 \text { Português } \\
77 \text { Espanhol }\end{array}$ \\
\hline SCIENCE DIRECT & $\begin{array}{l}\text { 7.626 Inglês } \\
3 \text { Português } \\
161 \text { Espanhol }\end{array}$ & $\begin{array}{l}2.125 \text { Inglês } \\
1 \text { Português } \\
30 \text { Espanhol }\end{array}$ & $\begin{array}{l}\text { 47.558 Inglês } \\
47 \text { Português } \\
1486 \text { Espanhol }\end{array}$ \\
\hline $\begin{array}{l}\text { Biblioteca Virtual em } \\
\text { saúde }\end{array}$ & $\begin{array}{l}5 \text { Inglês } \\
2 \text { Português } 0 \text { Espanhol }\end{array}$ & $\begin{array}{l}0 \text { Inglês } \\
0 \text { Português } \\
0 \text { Espanhol }\end{array}$ & $\begin{array}{l}10.208 \text { Inglês } \\
507 \text { Português } \\
310 \text { Espanhol }\end{array}$ \\
\hline SCIELO & $\begin{array}{l}5 \text { (inglês ) } \\
0 \text { em português } \\
1 \text { em espanhol }\end{array}$ & $\begin{array}{l}0 \text { (inglês ) } \\
0 \text { em português } \\
0 \text { em espanhol }\end{array}$ & $\begin{array}{l}138 \text { (inglês ) } \\
46 \text { em português } \\
78 \text { em espanhol }\end{array}$ \\
\hline Cochrane Library & $\begin{array}{l}790 \text { (inglês ) } \\
2 \text { em português } \\
0 \text { em espanhol }\end{array}$ & $\begin{array}{l}29 \text { (inglês ) } \\
29 \text { em português } \\
0 \text { em espanhol }\end{array}$ & $\begin{array}{l}8672 \text { (inglês ) } \\
29 \text { em português } \\
0 \text { em espanhol }\end{array}$ \\
\hline
\end{tabular}


Fonte: Autores (2020).

Nesses estudos coletados, foram triados ainda por período de publicação 2015 a 2020 (contendo raras excessoes que foram necessarias a inclusão para o contexto), onde foram retirados os artigos duplicados e os que eram. Bloqueados, onde os mesmos não foram utilizados, então não foram citados. Devido a toda essa triagem obtivemos um total de 22 artigos nas bases científicas já apresentadas (artigos nos 3 idiomas). Na Tabela 2 observamos os artigos que foram selecionados para a pesquisa.

Tabela 2. Artigos selecionados:

\begin{tabular}{|c|c|c|c|}
\hline & Autores & Artigos & Ano \\
\hline 1 & OLIVEIRA, & $\begin{array}{l}\text { Incidence of antiretroviral adverse drug reactions in pregnant women } \\
\text { in two referral centers for HIV prevention of mother-to-child- } \\
\text { transmission care and research in Rio de Janeiro, Brazil }\end{array}$ & 2014 \\
\hline 2 & ALMEIDA & $\begin{array}{l}\text { Influence of the antiretroviral therapy on the growth pattern of children } \\
\text { and adolescents living with HIV/AIDS }\end{array}$ & 2019 \\
\hline 3 & BRITES & $\begin{array}{l}\text { Use of new antiretroviral drugs and classes iBahia, Brazil: a real life } \\
\text { experience on salvagherapy of AIDS patients }\end{array}$ & 2015 \\
\hline 4 & SILVA, AM et al. & Real-world evidence in antiretroviral therapy: drugsafety & 2020 \\
\hline 5 & SOARESA, D S. & $\begin{array}{l}\text { Acute kidney injury in HIV-infected children: comparison of patients } \\
\text { according to the use of highlyactive antiretroviral therapy }\end{array}$ & 2016 \\
\hline 6 & $\begin{array}{l}\text { PÉREZ, MM, MILIAN, } \\
\text { AJG. }\end{array}$ & $\begin{array}{l}\text { Reacciones adversas evitables graves por antivirales. Sistema Cubano de } \\
\text { Farmacovigilancia, 2008-2017 }\end{array}$ & 2019. \\
\hline 7 & $\begin{array}{lr}\text { OLIVEIRA, } & \text { AM, } \\
\text { COSTA, } & \text { LF, } \\
\text { FONSECA, CA. } & \\
\end{array}$ & $\begin{array}{l}\text { A farmáco-psiquiatria e as reações adversas no uso de anti-retrovirais } \\
\text { revista eletrônica de farmácia .Suplemento Vol } 3 \text { (2), 31-33, }\end{array}$ & 2006. \\
\hline 8 & $\begin{array}{l}\text { SUBIELA, } \quad \text { JD.; } \\
\text { DAPENA EB }\end{array}$ & $\begin{array}{l}\text { Cambio de la terapia antiretroviral de gran actividad asociada a reacciones } \\
\text { adversas medicamentosas en un centro especializado en Venezuela. }\end{array}$ & 2016. \\
\hline 9 & GUITART A, JEM, XV. & $\begin{array}{l}\text { Evaluación de la respuesta y seguridad a diferentes esquemas de } \\
\text { tratamiento antirretroviral en Colombia. }\end{array}$ & 2020 \\
\hline 10 & BRITES, C et al. & $\begin{array}{l}\text { Raltegravir versus lopinavir / ritonavir para tratamento de mulheres } \\
\text { grávidas infectadas com HIV com apresentação tardia. }\end{array}$ & 2020. \\
\hline 11 & $\begin{array}{lrr}\text { FABRA, } & \mathrm{R} & \mathrm{L} ; \\
\text { ACOSTA, } & \text { MCR. }\end{array}$ & $\begin{array}{l}\text { Vigilancia farmacológica con el uso de los antirretrovirales. Rev cubana } \\
\text { med v. } 46 \text { n. } 4 \text { Ciudad de la Habana oct.-dic. }\end{array}$ & 2007. \\
\hline 12 & ELZI, L. & Eventos adversos de raltegravir e dolutegravir. & 2020 \\
\hline 13 & - & $\begin{array}{l}\text { Adverse drug reactions of antiretroviral therapy in patients receiving } \\
\text { methadone substitution treatment. }\end{array}$ & 2014. \\
\hline 14 & $\begin{array}{l}\text { JAIN A, LIHITE RJ, } \\
\text { MANGALA } \\
\text { BARUAH SK. }\end{array}$ & $\begin{array}{l}\text { Um estudo sobre reações adversas a medicamentos à terapia antirretroviral } \\
\text { de primeira linha em pacientes infectados pelo HIV em um hospital } \\
\text { terciário no nordeste da Índia. }\end{array}$ & 2016. \\
\hline 15 & $\begin{array}{l}\text { HIRSCH } \\
\text { AA, COMPAN A, } \\
\text { LAWRENCE R H. et. } \\
\text { al. }\end{array}$ & $\begin{array}{l}\text { Estudo piloto para avaliar relatos subjetivos e objetivos de estudo dos } \\
\text { efeitos adversos da terapia antirretroviral para a infecção pelo HIV. Indian } \\
\text { Journal of Medical Specialties . }\end{array}$ & 2015. \\
\hline
\end{tabular}




\begin{tabular}{l|l|l|l|l}
\hline $\mathbf{1 6}$ & $\begin{array}{l}\text { AGUT, H.; BURREL; } \\
\text { BONNAFOUS, P.; } \\
\text { BOUTOLLEAU D }\end{array}$ & . Antivirales (a excepción del VIH y la hepatitis). & 2020. \\
\hline $\mathbf{1 7}$ & PUCCI, RFM. & Adverse drug reactions. Elsevier. & 2020. \\
\hline $\mathbf{1 8}$ & KANTERS, S. & $\begin{array}{l}\text { Comparative efficacy and safety of first-line antiretroviral therapy for the } \\
\text { treatment of HIV infection: a systematic review and network meta- } \\
\text { analysis. }\end{array}$ & 2016. \\
\hline $\mathbf{1 9}$ & SIMO, S. & $\begin{array}{l}\text { Efeito da administração a longo prazo de medicamentos antirretrovirais } \\
\text { (Tenofovir e Nevirapina) na neuroinflamação e neuroplasticidade em } \\
\text { hipocampos de camundongos. }\end{array}$ & 2018. \\
\hline $\mathbf{2 0}$ & GENDELMAN, HE. & $\begin{array}{l}\text { A promessa de terapias anti-retrovirais de longa duração: da necessidade à } \\
\text { fabricação. }\end{array}$ & 2019. \\
\hline $\mathbf{2 2}$ & OLIVEIRA, MS. & $\begin{array}{l}\text { Farmacovigilância de medicamentos anti-retrovirais em gestantes } \\
\text { portadoras de HIV e em crianças espostas ao HIV durante a gestação em } \\
\text { uso de profilaxia da transmisão vertical: estudo piloto de incidência. }\end{array}$ & 2020. \\
\hline & $\begin{array}{l}\text { Terapia anti-retroviral isolada versus terapia anti-retroviral com uma } \\
\text { participantes com infecção recente por HIV (o estudo RIVER): um estudo } \\
\text { randomizado de fase 2. }\end{array}$ & 2020 \\
\hline
\end{tabular}

Fonte: Autores (2020).

\subsection{Efeitos colaterais em jovens, adultos e idosos}

O tratamento do vírus não pode curar por conta do acumulo persistente de células infectadas latentemente. Vários estudos pretendem descobrir a cura através dessas células, que se tornam mais simples para serem destruídas. O primeiro estudo randomizado, foi realizado em seis clínicas do Reino Unido, entre pacientes com a faixa etária de 18-60 anos (pacientes com o vírus). Esse estudo não obteve a eficácia desejada, porem auxilia futuras pesquisas (Lewin, 2020).

O uso da terapia para o tratamento da doença tem obtido eficácia, porem mesmo depois de anos, muitos registros de patologias neurocognitivas continuam a ser registrados nos portadores de HIV mesmo com baixa carga viral. De acordo com a pesquisa de Zulu, 2018, destacam que a terapia antirretrovirais altamente eficaz (TARV) é prejudicial ao cérebro e contribui diretamente as patologias neurocognitivas e também que a nevirapina promove processos inflamatórios (Zulu, 2018). De acordo com a pesquisa de Kanters, 2016 a substância TARV melhora a retenção, o que segundo ele, pode ser uma solução para países de baixa renda.

O tratamento da doença é importante, porém as reações adversas aos medicamentos podem diminuir a qualidade de vida dos portadores. De acordo com Fabra (2007), esses medicamentos apresentam muita toxicidade hematológica e também alterações hepáticas em alguns casos até mesmo letais. Também foram observadas muita toxicidade no sistema nervoso. De acordo com a OMS no final do ano de 2006, 42 milhoes de crianças e adultos haviam sido infectados pelo vírus e mais de 20 milhões haviam falecido por conta do vírus. As pesquisas tentam melhorar essa qualidade de vida correlacionando fármacos e criando tentativas para a criação de novo fármaco ou ainda a descoberta de uma possível vacina (Fabra et al, 2007).

A pesquisa a efeitos aos medicamentos é importante, atualmente $25 \%$ dos pacientes com HIV abandonam o tratamento por conta de falha do tratamento, efeitos tóxicos nos primeiros meses de terapia. Em uma pesquisa realizada em um hospital terciário de Mumbai mostrou que o efeito mais comum que foi registrado foi a anemia (17,5\%). Os profissionais devem observar essas reações adversas e tentarem minimizá-las para uma vida melhor (Dekate, 2020). 
Os medicamentos antirretrovirais se forem administrados por via parenteral de longa ação, são menos tóxicos e reduzem a resistência viral. Essas formulações de pró-droga aumenta a meia vida, facilitando a entrada e retenção da droga, distribuindo a droga com mais facilidade no tecido. Esses medicamentos transformam a morte certa pelo vírus, em possível tratamento podendo levar a uma vida mais duradoura. Por conta disso, pesquisas são realizadas todos os dias para redução desses efeitos e criação de medicamentos mais eficazes para seu tratamento até mesmo estudando a possível cura da doença (Kevadiya, 2019).

A formulação de pró-droga dos antirretrovirais demonstram grandes melhoras para absorção nas células e tecidos, nos intervalos de dosagem e nos volumes por nanossupressão. Essas novas formulas também suprimem cargas virais com o objetivo de eliminação do vírus, quando combinados com outras modalidades (Ibidem).

Com o avanço nas pesquisas podemos observar a combinação de medicamentos antiretrovirais com substâncias como a metadona. Essas combinações pretendem prolongar a vida dos portadores. Porém os seus efeitos colaterais são mais severos quando associados, sendo assim piorando a qualidade de vida dos pacientes. Os sintomas mais frequentes são: perda de apetite, constipação, tosse, rinite, insônia, libido e diminuição do nível de hemoglobina (Elni, 2020).

Em um estudo realizado no estado de Assam na India, demonstra que nessa região existem poucos casos registrados de reações adversas aos medicamentos de primeira linha: zidovudina, lamivudina, nevirapina, efavirenz, temofovir e estavudina. Nessa pesquisa os pacientes que utilizaram o efavirenz demonstraram distúrbios do sistema nervoso e distúrbios gastrointestinais, seguido de vômito, vertigem com poucos relatos. Nos demais medicamentos foram observados vômito e vertigem (Rajesh, 2016).

A pesquisa de Elni demonstrou efeitos neurológicos nos pacientes administrados com dolutegravir mais TARV, quanto ao raltegravir a toxicidade foi bem menor (Elni, 2020).

O fármaco afavirenz tem um bom resultado no tratamento da doença, entretanto esta relacionados frequentemente a eventos adversos como efeitos neuropsiquiatricos, nervosismo, depressão, ansiedade, tontura distúrbios do sono. Tem uma resposta rápida em crianças, mas possui os mesmos efeitos colaterais (Dalwadi, 2018).

Os dados sobre efeitos colaterais aos antirretrovirais são poucos registradas, por isso é muito difícil dividir entre jovens ou adultos que são afetados. Não foram observados diferença na gravidade de acordo com as faixas etárias (Hirsch et al, 2020).

A pesquisa de Fabra em 2007 mostra que os pacientes tratados com os medicamentos de primeira linha 1A, 1B e 1C, possuíram menos sintomas, porém alguns de forma grave e outros obtiveram pela utilização por longo prazo. Também ocorreram efeitos secundários menores, aos que utilizaram 1A e 1B, como: manifestações de efeitos neurológicos, gastrointestinais, dor de cabeça, diarreia, vômito e náuseas. Outros fatores foram observados como a síndrome de Stevens Johnson, reação foi observada por usuário de nevirapine. Também foram registrados necrose de epiderme produzida por efavirenz (como se fosse grande queimadura). Já nesse estudo não foram observados neutropenia severa.

Brites afirma que, o uso de medicamentos criados recentemente torna mais fácil o tratamento desses pacientes, principalmente quando se trata de cepas resistentes, permitindo uma maior qualidade de vida sem muitas anormalidades significativas, tornando-os mais seguros para serem utilizados (Brites et al, 2015).

Países como Portugal relatam a importância da notificação e que o não preenchimento prejudica no acompanhamento da doença. No país, os maiores efeitos adversos encontrados são: problemas de pele e problemas no sistema nervoso o que não diferencia muito dos artigos publicados no Brasil (Silva et al, 2020).

\subsection{Efeitos colaterais em gestantes}

As utilizações dos tratamentos antirretrovirais na gravidez são muito importantes pra evitar transmissão vertical. Mesmo assim, existem muitos efeitos colaterais observados em gestantes como: anemia, trombocitopenia, alergia, alterações em testes de função hepática, dislipidemia e diabetes, porém isso está relacionado ao uso de TARV. A utilização de nevirapina, nelfinavir e atazanavir influenciaram patologias hepáticas, já o uso de lopinavir gerou alterações na glicemia. Mesmo com esses efeitos 
colaterais relatados, eles possuem grande eficácia no tratamento e possui baixa gravidade nas alterações ocorridas (Delicio, 2020).

Existem poucos estudos publicados atualmente sobre a segurança de medicamentos usados para prevenção da transmissão durante o parto, principalmente em português. Geralmente gestantes que já estão tomando o medicamento desde a gravidez não sentem efeitos colaterais, porém as gestantes que iniciaram a tratamento já grávidas, tiveram vários tipos de efeitos como: cólicas, diarréia, náuseas e vômitos; cutânea, incluindo prurido e exantema; anemia; e hepatite. Havendo também complicações no parto como prematuros ou até mesmo os bebês possuindo baixo peso no nascimento ou malformações congênitas. Outros problemas foram observados nos exames hematológicos dos fetos como anemia, neutropenia ou plaquetopenia, houve também complicações hepáticas, exantema, dentre outros (Oliveira et al, 2007).

De acordo com a pesquisa realizada por Brites disponibilizada em 2020 observamos que de dois antiretrovirais utilizados para gestantes: o raltegravir e o lopinavir / ritonavir, o segundo obteve maior supressão virologica, onde também a maioria dos efeitos adversos de forma leve, a maioria problemas intestinais.

A toxicidade desses medicamentos varia pelo tempo de consumo, as classes e grupos dos fármacos, dentre outros fatores, em uma pesquisa realizada por Food and Drug Administration. em janeiro de 2005, mostrou que as gestantes possuem muito mais toxicidade nos hapatócitos (Fabra et al, 2007).

\subsection{Efeitos colaterais em pré-natal e lactente, infância e adolescência}

O vírus do HIV também contamina as crianças por todo o mundo, possuindo um número alarmante de infectados. Onde a principal forma de contaminação ocorre pela transmissão vertical, por isso a grande importância da saúde pública, para que possamos obter mudanças principalmente em países de baixa renda. No ano de 2017 no mundo 51\% dos atingidos foram mulheres, com idade de 15 a 24 anos. Em homens 44\%, sendo na mesma faixa etária de idade. O Brasil possui quase a metade de casos do cenário latino-americano (Almeida et al,2018).

Nas crianças essa doença é quase imperceptível, a maioria inicia a manifestação clínica a partir dos 5 anos de idade. Essas crianças apresentam alterações endócrinas, alterações tiroidianas, lipodistrofias, deslipidemias, resistência à insulina e hormônio de crescimento. No pré-natal e lactente possuem uma maior demora ao ganho de peso. Na infância necessitam de suplementos, por terem uma maior dificuldade de crescimento e ganho de gordura. Na adolescência demoram mais para o início do período purberal. As crianças ou adolescentes que possuem desnutrição apresentam maior morbidade. Também podem ser utilizados antibióticos como profiláticos para redução pneumocistose (Ibidem).

De acordo com Oliveira, 2013 esses medicamentos não causam má formação do feto e nem que nasçam prematuros.

De acordo com pesquisa realizada no estado do Ceara com crianças na faixa etária de 5 anos, observou-se grandes efeitos nefrotoxicos, principalmente com a utilização de tenofovir; seguido por presença de hipocalemia e também acidose láctica (Soares, 2016).

\section{Conclusão}

Independente da faixa etária dos portadores ou gestantes com o vírus, mostramos nessa pesquisa que os tratamentos utilizados para a doença ainda apresentam muitos efeitos colaterais onde na maioria deles são efeitos neurológicos, mas também foram observados problemas hepáticos, problemas renais, hematológicos, diabetes, perda de apetite, constipação, tosse, rinite, insônia, libido, anemia, problemas hormonais, problemas inflamatórios, grastrointestinais, seguido de vômito, vertigem ou até mesmo Sindrome de Stevens Johnson (problemas no tecido epitelial).

Com isso, mostramos a importância de mais pesquisas para a diminuição dos efeitos adversos, ou a criação de novos fármacos com efeitos mais leves ou até mesmo a criação de uma possível vacina. 
Mesmo sendo sintomas leves, podem levar a uma diminuição da qualidade de vida do portador, por isso se faz necessário mais pesquisas sobre o tema, inclusive em nosso idioma, que como mostramos foi a minoria apresentada. Devemos lembrar que a qualidade de vida deles impacta diretamente a nossa sociedade, portanto a qualidade de vida desses pacientes importa a todos.

\section{Referências}

Agut, H., Burrel, S., Bonnafous, P., \& Boutolleau, D. (2017). Antivirales (a excepción del VIH y la hepatitis). EMC - Tratado de Medicina, 21 (1), 1-10.

Almeida, F. J., Kochi, C., \& Sáfadi, M. A. P. (2019). Influence of the antiretroviral therapy on the growth pattern of children and adolescents living with HIV/AIDS. J Pediatr (Rio J). 95 (1), 95-101.

Alochio, K. V. \& Gouvêa, M. V. (2016). Formação e prática de multiplicadores regionais em testesrápidos de hiv. https://webcache.googleusercontent.com/search?q=cache:ty4kS0x8xdkJ:https://periodicos.ufpe.br/revistas/revistaenfermagem/article/download/11020/12401 $+\& \mathrm{~cd}=1 \& \mathrm{hl}=\mathrm{ptBR} \& \mathrm{ct}=\mathrm{clnkgl}=\mathrm{br}$

Departamento de Doenças de Condições Crônicas e Infecções Sexualmente Transmissíveis. Ministério da Saúde. Manual Técnico para Diagnóstico da Infecção pelo HIV em Adultos e Crianças. http://www.aids.gov.br/ptbr/node/57787.

Ministério da Saúde. O que você precisa saber - Sobre a Doença: coronavirus (covid-19). Acesso à informação. https://coronavirus.saude.gov.br/; https://coronavirus.saude.gov.br/sobre-a-doenca\#o-que-ecovid.

Brites, C. (2020). Raltegravir versus lopinavir / ritonavir para tratamento de mulheres grávidas infectadas com HIV com apresentação tardia. Elsevier.

Brites, C., Nóbrega, I., \& Martins, E. N. (2015). Use of new antiretroviral drugs and classes iBahia, Brazil: a real-life experience on salvagherapy of AIDS patients. braz. j. infect. dis. 19 (5), 529-532.

Carneiro, S. A. (2020). Efeitos Adversos da Terapia Antirretroviral. Médica Pediatra CTR DIP Orestes Diniz /PBH e HC/Ebserh Pós-Graduação em Ciências da Saúde: Saúde da Criança e do Adolescente (UFMG). http://www.smp.org.br/arquivos/site/pediatras/comunicados/efeitos-adversosdosantirretrovirais-pdf.pdf.

Cooper, H. M. Scientific guidelines for conducting integrative research reviews. (1982). Review of Educational Research. 52 (2), 291-302. https://journals.sagepub.com/doi/10.3102/00346543052002291.

Elzi, L. Eventos adversos de raltegravir e dolutegravir. Elsevier

Fabra, R. L., \& Acosta, M. C. R. (2007). Vigilancia farmacológica con el uso de los antirretrovirales. Rev cubana med. 46 (4).

Fidler, S. em participantes com infecção recente por HIV (o estudo RIVER): um estudo randomizado de fase 2. The Lancet, 395 (0227), 844-846.

Gendelman, H. E. (2019). A promessa de terapias anti-retrovirais de longa duração: da necessidade à fabricação. Elsevier.

Hirsch, A. A., Compan, A., Lawrence, R. H., et. al. (2015). Estudo dos efeitos adversos da terapia antirretroviral para a infecção pelo HIV. Indian Journal of Medical Specialties.

Huffpost. Brasil. (2017). Tratamento do HIV/Aids: 'Hoje basta um comprimido por dia. https://www.huffpostbrasil.com/jovemsoropositivo/aids-hoje-basta-umcomprimido-por dia_b_6573254.html.

Jain, A., Lihite R. J., Mangala L., \& Baruah, S. K. (2016). Um estudo sobre reações adversas a medicamentos à terapia antirretroviral de primeira linha em pacientes infectados pelo HIV em um hospital terciário no nordeste da Índia. Sociedade Polonesa de Pesquisa em AIDS.

Kanters, S. (2016). Comparative efficacy and safety of first-line antiretroviral therapy for the treatment of HIV infection: a systematic review and network metaanalysis. Elsevier.

Loj, P., Gąsiorowski, J., Łapiński, Ł., Zubkiewicz-Zarębska, A., \& Knysz, B. (2014). Adverse drug reactions of antiretroviral therapy in patients receiving methadone substitution treatment. Elsevier. 13 (1), 14-17.

Machado, J. E., A. \& Vidal, X. G. (2013). Evaluación de la respuesta y seguridad a diferentes esquemas de tratamiento antirretroviral en Colombia. Investig. andina. $15(27), 770-783$.

Oliveira, A. M., Costa, L. F., \& Fonseca, C. A. (2006). A farmáco-psiquiatria e as reações adversas no uso de anti-retrovirais. Revista Eletrônica de Farmácia e Suplemento. 3 (2), 31-33.

Oliveira, B. O., \& Medeiros, L. H. Q. (2018). Relação do estado nutricional e parâmetros clínicos com a percepção da qualidade de vida em pacien tes que vivem com Hiv/aids acompanhados no ambulatório de um hospital de Pernambuco. Tese. http://tcc.fps.edu.br/bitstream/fpsrep o/217/1/RELA\%C3\%87\%C3\%83O\%20DO\%2ESTADO\%20NUTRICIONAL\%20E\%20PAR\%C3\%82METROS\%20CL\%C3\%8DNICO\%20COM\%20A\%20 PERCEP\%C3\%87\%C3\%83O.pdf.

Oliveira, M. S. (2007). Farmacovigilância de medicamentos anti-retrovirais em gestantes portadoras de HIV e em crianças espostas ao HIV durante a gestação em uso de profilaxia da transmissão vertical: estudo piloto de incidência. Tese. 123.

Oliveira, M. S., Friedman, R. K., Veloso, V. G., Cunha, C. B., Pilotto, J. H., Marins, L. M. S. et al. (2014). Incidence of antiretroviral adverse drug reactions in pregnant women in two referral centers for HIV prevention of mother-to-child-transmission care and research in Rio de Janeiro. Brazilian Journal of Infectious Diseases. 18 (4): 372-378. 
Research, Society and Development, v. 10, n. 3, e50210313308, 2021

(CC BY 4.0) | ISSN 2525-3409 | DOI: http://dx.doi.org/10.33448/rsd-v10i3.13308

Pereira, A. S., Shitsuka, D. M., Parreira, F. J., \& Shitsuka, R. (2018). Metodologia da pesquisa científica. UFSM.

Pereira, V. S. (2012). A emergência de novidades metodológicas no campo virtual: uma análise dos estudos no ciberespaço. In Simpósio nacional da Associação Brasileira de cibercultura. Anais do ABCiber. 6, 1-7. http://gitsufba.net/anais/wp-content/uploads/2013/09/n1_emergencia_44913.pdf.

Pérez, M. M., \& Milian, A. J. G. (2019). Reacciones adversas evitables graves por antivirales. Sistema Cubano de Farmacovigilancia, 2008-2017. Horizonte sanitário. 17 (1).

Primeira, M. R., Santos, W. M., Paula, C. C., \& Padoin, S. M. (2020). Qualidade de vida, adesão e indicadores clínicos em pessoas vivendo com HIV. Acta Paul Enferm. 33.

Pucci, R. F. M. (2020). Adverse drug reactions. Elsevier.

Silva, A. M., Pereira, M., Dias, C. C., Ventura, Â., \& Sousa-Pinto, B. (2020). Real-world evidence in antiretroviral therapy: drug safety data. The Brazilian journal of infectious diseases: an official publication of the Brazilian Society of Infectious Diseases, 24(3), 271-272.

Simo, S. (2018). Efeito da administração a longo prazo de medicamentos antirretrovirais (Tenofovir e Nevirapina) na neuroinflamação e neuropla sticidade em hipocampos de camundongos.Elsevier.

Soares, D. S. (2016). Acute kidney injury in HIV-infected children: comparison of patients according to the use of highlyactive antiretroviral therapy. $J$ Pediatr. $92(6), 631-637$.

Souza, H. C., Mota, M. R., Alves, A. R., Lima, F. D., Chaves, S. N., Dantas, R. A. E., Abdelmur, S. B. M. \& Mota, A. P. V. S. (2019). Analyis of compliance to antirretroviral treatment among patients with hiv/aids. Rev Bras Enferm. 72 (5), 1295-303.

Subiela, J. D., \& Dapena E. B. (2016). Cambio de la terapia antiretroviral de gran actividad asociada a reacciones adversas medicamentosas en un centro especializado en Venezuela. Invest Clin. 57 (1), 3 -12.

Suto, C. S. S., Paiva, M. S., Oliveira, J. F., Rodrigues, A. S., Oliveira, D. S., \& Porcino, C. A. (2017). O cuidado à Síndrome da Imunodeficiência Adquirida na contemporaneidade: compreendendo o olhar de profissionais. Revista da Rede de Enfermagem do Nordeste. https://www.redalyc.org/articulo.oa?id=324054583011.

Unaids - Programa Conjunto das Nações Unidas sobre HIV/Aids. (2020). HIV e COVID 19: O que as pessoas que vivem com HIV precisam saber sobre HIV e COVID-19. https://unaids.org.br/2020/04/o-que-as-pessoas-que-vivem-com-hiv-precisam-saber-sobrehive-covid-19/. 